\title{
Uso da realidade aumentada como ferramenta auxiliar ao vídeo educacional no ensino presencial e à distância
}

\section{Use of augmented reality as a tool to assist educational video in the classroom and distance teach}

\author{
Eduardo Luis Schneider, André Grassi, Leonardo Piccoli, Maria \\ Isabel Timm \\ Centro Nacional de Supercomputação/CESUP, Universidade Federal do \\ Rio Grande do Sul/UFRGS \\ Av. Osvaldo Aranha, 99 CEP 90035-190, Porto Alegre, RS \\ eduardo@cesup.ufrgs.br
}

\section{Resumo}

Neste trabalho é apresentado um estudo sobre o uso da realidade aumentada como ferramenta auxiliar ao vídeo educacional. Foram feitos experimentos dentro da estrutura já existente no CESUP/UFRGS (Centro de Supercomputação da Universidade Federal do Rio Grande do Sul) com câmeras controladas com joystick e através de uma mesa de apresentação de documentos. Foi produzido um vídeo de caráter exploratório e demonstrativo sobre o uso da RA (Realidade Aumentada) como tecnologia educacional para a criação de uma rede adicional para o Supercomputador do CESUP/UFRGS. Os experimentos mostram que o emprego da RA na área de educação e treinamento apresenta um grande potencial de auxiliar o processo de construção do conhecimento dos usuários.

Palavras-Chave: realidade aumentada, tecnologias educacionais, educação à distância, vídeo educacional.

\begin{abstract}
This paper presents a study on the use of augmented reality as a tool to assist educational video. Experiments were done within the existing structure in CESUP/UFRGS with cameras controlled by joystick and through a table for production of documents. It was produced a video of exploratory and demonstration on the use of AR (Augmented Reality) as educational technology to create an additional network for Supercomputer in CESUP/UFRGS. The experiments showed that the use of $A R$ in education and training has great potential to assist the process of constructing knowledge of users.
\end{abstract}

Keywords: augmented reality, educational technologies, distance education, educational video. 


\section{Introdução}

O crescente aumento na complexidade imposta por tarefas em diversas áreas da Ciência está exigindo cada vez mais do homem, frente às suas limitações naturais. Da mesma maneira, o número de trabalhos que incorporaram o computador para o seu desenvolvimento vem crescendo e aumentando, também, a dependência do homem em relação à máquina. Para lidar com esta realidade, surge a necessidade da criação de ambientes mais interativos, que permitam que o homem minimize possíveis dificuldades na manipulação do computador e, por conseguinte, execute seu trabalho de modo mais eficiente e satisfatório. Com o uso de novas tecnologias, as atividades e as práticas de ensino se tornam mais favoráveis para o aprendizado, influenciando diretamente em sua qualidade [1].

Através da evolução das tecnologias de mistura da Realidade Virtual (RV) com o mundo real, é possível colaborar com as diversas áreas do conhecimento, tanto das ciências humanas quanto das exatas. Nas engenharias, por exemplo, onde são buscadas soluções para muitos processos industriais de intensa complexidade e que demandam o uso de ferramentas de visualização dos seus sistemas, técnicas de visualização como a RV e a Realidade Aumentada (RA) se apresentam como poderosa ferramenta para visualização tridimensional. O suporte aos processos industriais de produção pode se dar através da sobreposição de objetos virtuais gerados pelo computador ao ambiente real, como partes funcionando em tempo real de um equipamento que precise ser monitorado.

Este trabalho visa desenvolver e utilizar a RA como ferramenta de suporte ao Núcleo de Pesquisa e Desenvolvimento de Tecnologias Educacionais e EAD do Centro Nacional de Supercomputação - CESUP, da Universidade Federal do Rio Grande do Sul - UFRGS. O Centro oferece Processamento de Alto Desempenho (PAD) a usuários e pesquisadores que necessitam resolver problemas avançados e realizar simulações, tanto em ciências básicas como em aplicadas aos mais diversos campos [2]. Um dos serviços prestados é o treinamento dos pesquisadores para o uso do PAD. O Núcleo de Pesquisa e Desenvolvimento de Tecnologias Educacionais e EAD se encarrega dessa tarefa e também mantém parcerias na pesquisa e uso de tecnologias educacionais.

\section{Realidade Aumentada}

A Realidade Aumentada (RA) permite representar dados e informações que não estão diretamente disponíveis ou detectáveis apenas pelos sentidos humanos, e seu emprego possibilita aumento dessa percepção. Assim, pode auxiliar na melhoria da interação com o mundo real e, consequentemente, aumentar a produtividade na realização dos mais variados tipos de tarefas. Entre as áreas de aplicação que já vêm sendo exploradas, podem ser citadas: publicidade, jogos, entretenimento, ilusionismo, indústria automobilística, militar, turismo, preservação histórica, mercado imobiliário, medicina, comunicação, movimentação de robôs, manutenção de equipamentos e educação.

Sabe-se que a simulação da realidade está longe de ser comparada ao mundo real conhecido. A mescla de ambientes virtuais e reais apareceu como uma alternativa: a adição de objetos virtuais a uma cena real. Essa área é chamada de Realidade Misturada (RM) e trabalha com aplicações em que ambientes virtuais e reais são sobrepostos, permitindo aos usuários manter o senso de presença no ambiente onde se encontram, continuando a ver ou sentir os elementos do ambiente real [3, 4]. Segundo Milgram (1994), na RM, a visão do mundo real é combinada com o modo de visão do 
Ambiente Virtual (AV), e as transições entre esses dois pilares dividem a RM em duas sub-áreas: a Realidade Aumentada (RA) e a Virtualidade Aumentada (VA), conforme ilustra a Figura 1. Na VA, o ambiente principal ou predominante é o mundo virtual. Na RA, o que predomina é o mundo real.

$\mathrm{Na}$ VA, o AV é realçado por meio do acréscimo de imagens, elementos ou sensações advindas do mundo real, fazendo com que o usuário possa ver seu próprio corpo, por exemplo, como algo presente nas simulações geradas por computador. Outra possibilidade seria inserir uma mobília real em um ambiente virtual criado [8,9].

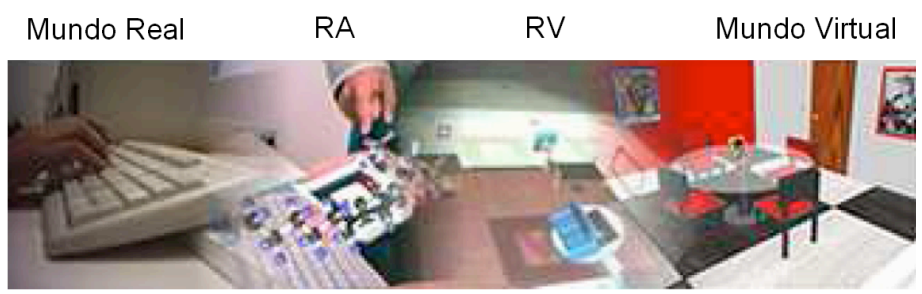

Figura 1 - Ambiente de Realidade Misturada. Adaptado de Milgram, 1994.

Já a RA pode ser definida como a sobreposição de objetos virtuais ao mundo real, por meio de um dispositivo tecnológico, melhorando ou aumentando a visão do usuário $[5,6,7]$. Cabe ressaltar que, neste caso, os objetos virtuais são trazidos para o espaço do usuário, onde ele tem segurança e sabe como interagir, sem a necessidade de treinamento. Além disso, o usuário consegue manipular os objetos reais e virtuais do ambiente misturado com o uso das próprias mãos. A RA pode ser empregada para a capacitação de funcionários, inclusive em serviços de risco para a aplicação ou até para o próprio funcionário. $\mathrm{O}$ usuário pode obter informações a respeito de um determinado equipamento danificado e ser guiado para a solução do problema a partir da adição de informações virtuais ao ambiente real onde o equipamento está.

$\mathrm{O}$ rastreamento, em ambientes de RV e RA, tem a função de identificar a posição do usuário ou de algo atrelado a ele, e permite o controle de posicionamento em ambientes virtuais ou aumentados. Diferentes dispositivos de rastreamento foram desenvolvidos, usando princípios mecânicos, magnéticos, de ultra-som, ótico, etc. $\mathrm{O}$ rastreamento ótico, junto com a popularização da webcam e o aumento do poder de processamento dos microcomputadores, está fazendo com que a RA esteja se apresentando como uma possibilidade concreta de vir a ser a próxima geração de interface popular.

\subsection{Bibliotecas Gráficas}

Para o desenvolvimento de aplicações de RA é necessário utilizar algumas bibliotecas gráficas. A mais conhecida atualmente é o ARToolKit que, além de apresentar resultados adequados, é gratuita e de código aberto [10]. Existem outras, como o ARTag [11, 12], que estão sendo desenvolvidas com propósitos comerciais.

O ARToolKit é um software desenvolvido pelo Dr. Hirozaku Kato da Universidade de Osaka, no Japão, e apoiado pelo Human Interface Technology Laboratory da Universidade de Washington e de Canterbury na Nova Zelândia. Foi projetado para o desenvolvimento de aplicativos de RA, provendo técnicas de visão computacional para calcular a posição e a orientação de marcadores impressos e capturados por uma câmera digital ou um Head Mounted Display (HMD), de forma que os marcadores venham a ser revestidos por objetos virtuais 3D. Utiliza-se de técnicas de visão computacional para calcular o ponto de vista da câmera em relação a um marcador existente no mundo real. Cada quadro de imagem do ambiente capturado é binarizado para facilitar o reconhecimento e a posição espacial do marcador. O desenho no interior V. $9 \mathrm{~N}^{\mathrm{o}} 1$, julho, 2011 
do marcador reconhecido é comparado com marcadores pré-definidos, e assim o marcador é sobreposto por uma imagem. A imagem gerada pode ser de um objeto desenvolvido em bibliotecas gráficas como OpenGL, ou importado em linguagem VRML (Virtual Reality Modeling Language). O VRML é uma linguagem para modelagem em RV. Seu objetivo é a criação e representação de mundos tridimensionais e objetos interativos através da WWW (World Wide Web) na internet. Foi apresentada pela primeira vez em 1994 durante a conferência sobre WWW com o propósito de atingir tais objetivos sem necessitar de uma rede de alta velocidade para dar suporte às atividades [13].

Para facilitar o desenvolvimento de aplicações colaborativas de realidade aumentada para usuários não especialistas em computação, foi desenvolvido o Sacra, que é uma ferramenta de autoria, onde as atividades de configuração podem realizadas utilizando pastas e arquivos de texto, elaborados com o Bloco de Notas do Windows ou equivalente [14].

\subsection{Realidade Aumentada, Ensino à Distância e Manutenção}

Ambientes de Realidade Virtual e Aumentada (RVA) implementados em rede ampliam em muito o potencial de aplicações educacionais. O estudo da RA em ambientes colaborativos proporciona compartilhamento de objetos virtuais no espaço real, de modo que todos os usuários possam ter a mesma sensação de espaço, presença e tempo [15].

Um interessante estudo sobre máquinas elétricas auxiliado por técnicas de RV é mostrado em [16]. Outro estudo relacionado com RA foi sobre o desenvolvimento de uma interface gráfica para o ARToolKit, com aplicação na área educacional [17]. Em [18] são exemplificados mais alguns trabalhos sobre essa abordagem, como o de uma aplicação para treinamento industrial colaborativo baseado em RA distribuída. Também é apresentado o desenvolvimento de um Ambiente Virtual de Aprendizagem, denominado AVADPT, que contém módulos de realidade virtual e realidade aumentada. $\mathrm{O}$ ambiente criado é adaptativo, de acordo com o grau de familiaridade do professor com a tecnologia, e pode ser usado separadamente ou em conjunto com outros ambientes educacionais, como o Moodle.

Uma interessante área de treinamento é a de manutenção de equipamentos, principalmente em processos industriais, pois nesses casos os ambientes tradicionais de treinamento apresentam limitações e alto custo [19]. Tecnologias RVA podem permitir a visualização e manipulação de componentes de difícil acesso, além de oferecer um suporte nas operações de montagem e desmontagem de equipamentos, nem sempre previstas em manuais. A utilização de interfaces para exibir as informações de suporte à manutenção cria uma maior interatividade do usuário com o equipamento em que é necessário realizar alguma intervenção. Alguns requisitos a respeito de treinamento virtual são apresentados em [20,21]

Uma aplicação interessante foi desenvolvida por pesquisadores do grupo Steve Feiner, da Universidade de Columbia. Ela ajuda usuários no processo de manutenção de uma impressora laser [22]. Este projeto usa rastreadores de posição localizar a parte dessa impressora na qual é necessário realizar uma operação. Além disso, assinala a presença ou não de algum objeto obstruindo o caminho de uma determinada ação que, concluída, eliminaria a necessidade do modelo virtual.

Para esses propósitos, equipamentos de visualização como TabletPC, PDA (Personal Digital Assistant), HMD e outros podem ser usados [23]. O usuário também pode receber informações do sistema de manutenção, como gráficos $2 \mathrm{D}$, modelos $3 \mathrm{D}$ e guias 
textuais de informações de manutenção. Outra possibilidade é a interação por comando de voz, especificando-se os componentes do equipamento e o tipo de informação que se deseja visualizar [24]. Diversos outros trabalhos poderiam ser citados aqui. Contudo, não foi objetivo deste trabalho consolidar ou contrapor o estado da arte na área de RA, mas sim mostrar um direcionamento que está sendo dado no CESUP/UFRGS.

\section{Infra-estrutura de EAD e Multimídia do CESUP/UFRGS}

Desde sua implantação em 2007, a infra-estrutura de produção de aulas à distância e multimídia educacional do CESUP/UFRGS tem servido ao treinamento dos usuários do cluster (conjunto de computadores com sistema operacional distribuído) Sun Fire, mas igualmente à pesquisa e parcerias na área de tecnologias educacionais. Conforme descrito por em trabalho anterior [25], o conjunto pretende ser um "sistema permanentemente instalado (plug-and-play) de produção, edição e transmissão de eventos interativos", priorizando "a criação de condições para ministrar cursos que demandem alta exigência de visualização, ou que possam ser beneficiados pelos recursos audiovisuais em multimídia, para apoio à compreensão de conteúdos exigentes e complexos, como a computação de alto desempenho, e outras áreas científicas e tecnológicas, como engenharia e medicina".

A infra-estrutura montada no Núcleo de Pesquisa e Desenvolvimento de Tecnologias Educacionais e EAD do CESUP dispõe de sala de aula (e estúdio de gravação) com isolamento acústico e iluminação adequada; duas câmeras Sony EVI-D100 com controle remoto por joystick para captação do ambiente; uma mesa de apresentação de documentos Elmo HV-5100XG, dotada de câmera de vídeo própria e iluminadores orientados a $45^{\circ}$; um computador de uso do professor com saídas de vídeo e áudio conectadas ao sistema; e uma ilha de captação/edição com software/placa de captura NewTek Video Toaster, que possibilita escolher, em tempo real, entre as quatro fontes de vídeo mencionadas. Todos esses recursos estão instalados de forma permanente, tendo em vista a agilidade e funcionalidade, mas também permitindo a integração entre si e com outras tecnologias a serem acrescentadas. É assim que se está experimentando a Realidade Aumentada. Através da técnica, o professor cuja aula está sendo gravada e/ou transmitida pode apresentar uma visualização 2D ou 3D de determinado objeto, componente ou cena, como se estivesse em sua mão, da mesma forma que faria com um livro aberto em que se apresentasse uma ilustração. Neste caso, porém, a imagem é integrada com a referida estrutura de captação e edição de vídeo, podendo ser visualizada em detalhe por cada aluno, presencial e/ou à distância, em tempo real ou através de acesso posterior ao acervo de vídeo-aulas.

Pesquisa-se também a possibilidade de utilização remota da Realidade Aumentada, pelos alunos à distância: o acesso a determinado conteúdo de vídeo, áudio e/ou 3D com o próprio computador do estudante. Desta forma, se experimentaria laboratórios virtuais de baixo custo e sem grande estrutura remota, conforme descrito por Forte e outros [26]: "o usuário geralmente observa a ocorrência de eventos a partir da tela do computador, sem a necessidade de se munir de dispositivos especiais de visualização, e interage com os objetos virtuais com o auxílio de marcadores tangíveis, sem a necessidade, também, de dispositivos especiais para a tarefa".

\section{Utilização da RA como Suporte a Criação de uma VLAN}

A integração da RA à estrutura já existente no CESUP/UFRGS está em fase experimental, através da produção de um vídeo nos mesmos moldes das vídeo-aulas regulares geradas na instituição, mas com caráter exploratório e demonstrativo. $\mathrm{O}$ V. $9 \mathrm{~N}^{\circ} 1$, julho, 2011 
conteúdo didático dessa vídeo-aula é o procedimento de criação de uma rede adicional para o cluster. Neste estudo de caso, o professor demonstra como criar uma rede local virtual, denominada VLAN, através do switch (dispositivo que gerencia as redes para reencaminhar módulos - frames - entre os diversos nós). Dentro do cluster do CESUP há três redes geridas. Uma rede de alto desempenho, uma de gerência e outra de trabalho (normal). Para dividir a rede em segmentos menores, cada rede adicional pode ser criada primeiramente acessando-se a interface de configuração do switch e identificando que portas devem ser utilizadas.

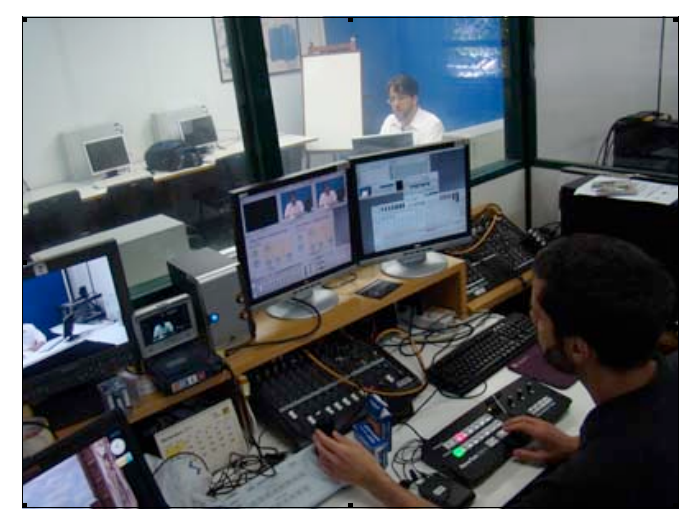

Figura 2 - Estúdio de gravação do CESUP/UFRGS.

O vídeo pretende apresentar o switch, seu funcionamento e seus componentes, para que o aluno seja capacitado a criar uma rede adicional para a máquina. A produção está sendo feita no estúdio de gravação do CESUP/UFRGS, com captação e edição em tempo real através da ilha de edição (Figura 2). Inicialmente, se produziu um teste de uso da tecnologia, com a utilização e demonstração da RA e dos outros recursos já utilizados regularmente.

$\mathrm{Na}$ primeira parte da vídeo-aula demonstrativa, o equipamento em questão foi mostrado através de sua imagem inserida em tela cheia no vídeo; e de sua imagem misturada eletronicamente com a do professor, de forma que este pudesse apontar fisicamente partes e componentes (Figura 3). Essa tecnologia, chamada de chroma-key, é conhecida por sua utilização em programas televisivos de previsão meteorológica, em que o apresentador é mostrado junto ao mapa. Este vídeo foi captado e editado nas instalações do CESUP/UFRGS, utilizando o equipamento já citado, e finalizado no software Adobe Premiere CS4. Está disponível para visualização no canal do YouTube do CESUP, onde também estão publicadas outras produções realizadas pelo Centro [27].

Num segundo momento, experimentou-se a RA (também abordada no vídeo acima citado), executada através do sistema Sacra versão 2010, disponível em [28]. Foram previamente impressos os marcadores, usando os arquivos .pdf disponibilizados e inserido um novo objeto 3D - uma chapa plana modelada com a linguagem VRML através de um arquivo texto com extensão .wrl. Sobre ela, foi aplicada como textura uma foto do switch em formato .jpg. Para elaboração dessa chapa, primeiramente definiu-se um objeto tridimensional (em VRML 2 chamado de Shapes) que em geral contém dois atributos, a Aparência e a Geometria. Na Aparência definiu-se a textura, no caso a imagem a ser exibida. Já na Geometria definiu-se um objeto em forma de cubo, com as dimensões do painel (largura 10, altura 0.01 e profundidade 7.5). 


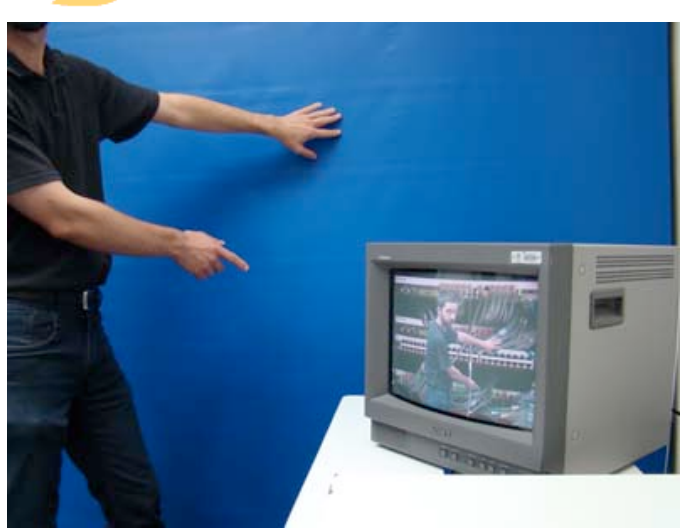

Figura 3 - Imagem do professor misturada eletronicamente com a do equipamento, através de chroma-key.

Para essa parte do experimento, um computador ASUS, dotado de webcam D-MAX $1.3 \mathrm{M}$, e executando a RA, foi conectado à ilha de captação e edição de vídeo, para receber o sinal que normalmente é gravado e/ou transmitido. As duas câmeras do estúdio de gravação foram utilizadas para capturar a imagem do professor segurando o marcador. Na imagem captada/gravada, aparece o professor segurando a fotografia do equipamento em questão, como se ele estivesse manipulando o próprio equipamento e seus componentes (Figura 4).

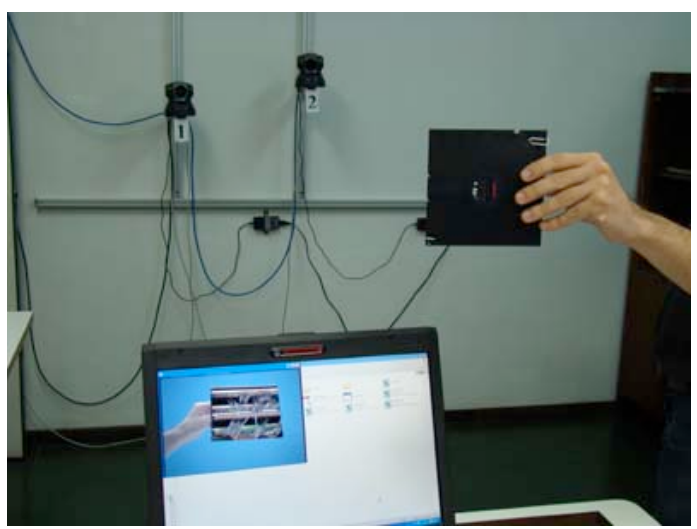

Figura 4 - Professor segurando o marcador para a captura por câmeras com controle remoto e a imagem do switch no monitor.

Uma variação dessa experiência foi feita através da mesa de apresentação de documentos. Nessa mesa, que normalmente serve para apresentar transparências ou pequenos objetos, foi colocado o marcador e, conforme a Figura 5, a imagem captada/gravada mostra o próprio equipamento em questão, como se estivesse sendo manipulado e apresentado pelas mãos do professor. 


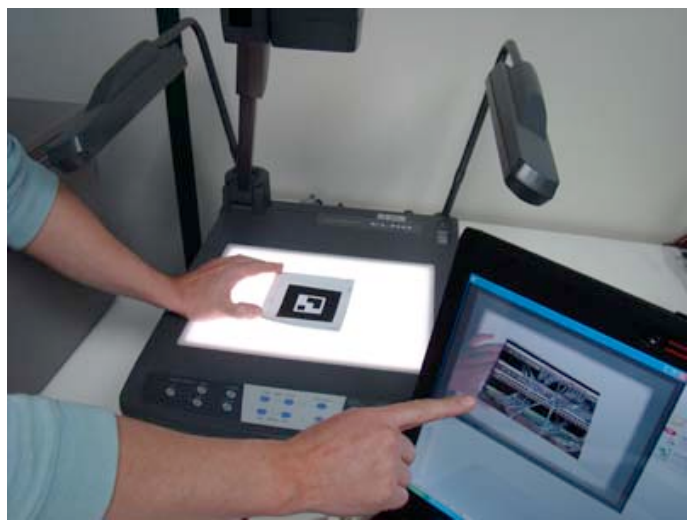

Figura 5 - Suporte a criação de uma rede adicional com a visualização das portas do switch através da RA.

Comparando a RA com as outras ferramentas, pôde-se verificar que em relação à mesa de apresentação de documentos, a RA reduz o custo de produção, já que não é necessário imprimir lâminas ou transparências de cada material que se queira colocar na mesa de apresentação para mostrar aos alunos. Comparando com a técnica de chromakey, notou-se que a RA permite uma melhor usabilidade já que com uma pequena variação no desenho dos marcadores é possível incluir pontos que podem funcionar como botões e uma vez que os mesmos sejam tocados, um outro objeto previamente associado o mesmo pode ser mostrado. Ou seja, também seria possível, por exemplo, apresentar uma modificação do mesmo indicando uma seqüência de uma operação de montagem, através da oclusão de um ponto que seria tapado por um dedo. Em todos os casos, a possibilidade de utilização de comando de voz através de um software de reconhecimento, como o Microsoft Speech, poderia permitir uma interação ainda maior.

Neste primeiro momento, foi experimentada a RA adicionada às demais tecnologias de apresentação já utilizadas no CESUP/UFRGS para ensino e treinamento. Pretendese dar continuidade a este trabalho estudando a inserção de objetos $3 \mathrm{D}$ e técnicas de comando de voz.

\section{Conclusão}

Neste trabalho foi feito um estudo sobre o uso da realidade aumentada como ferramenta auxiliar às demais tecnologias educacionais no ensino presencial e à distância. Foram feitos experimentos dentro da estrutura já existente no CESUP/UFRGS com câmeras controladas com joystick e através de uma mesa de apresentação de documentos. Foi produzido um vídeo de caráter exploratório e demonstrativo sobre o uso da RA como tecnologia educacional para a criação de uma rede adicional para o cluster do CESUP/UFRGS. Os experimentos mostraram que o emprego da RA na área de educação e treinamento apresenta um grande potencial de auxiliar o processo de construção do conhecimento dos usuários através da visualização e a manipulação de objetos virtuais modelados por computador no mundo real.

\section{Agradecimentos}

Ao CNPQ, aos colegas do CESUP, em especial ao técnico Lindolfo Meira e a diretora Denise Grüne Ewald, e ao professor Renato Ventura Bayan Henriques e demais colegas do Departamento de Engenharia Elétrica da UFRGS. 


\section{Referências}

[1] KLOPFER, E. Augmented Learning Research and design of móbile Educational games. Massachusetts Institute of Technology, 2008.

[2] [http://www.cesup.ufrgs.br/]. Visitado em 27/01/2011.

[3] HULF, R.; NEDEL, L.P.; OLIVEIRA, M.M; FREITAS, C.M.D.S. Usando Iluminação Baseada em Imagens na Geração de Ambientes de Realidade mista. In: Workshop sobre Realidade Aumentada, Piracicaba, Brasil, Maio, 2004.

[4] AZUMA, R.; BAILlOT, Y.; BEHRINGER, R.; FEINER, S.; JULIER, S.; MACINTYRE, B. Recent Advances in Augmented Reality. IEEE Computer Graphics \& Applications, v. 21, n. 6, p. 34-47, novembro/dezembro, 2001.

[5] BAJURA, M.; NEUMANN, U. Dynamic Registration Correction in Video-

Based Augmented Reality Systems. IEEE Computer Graphics \& Applications, v.15, n.5. p.52-60, 1995.

[6] AZUMA, R. T. A Survey of Augmented Reality. Presence: Teleoperators and Virtual Environments, v.6, n.4, p. 355-385, 1997.

[7] MILGRAM, P. et al. Augmented Reality: A Class of Displays on the RealityVirtuality Continuum. Telemanipulator and Telepresence Technologies, SPIE, V.2351, 1994.

[8] SISCOUTO, R.A.; TORI, R. AVTC-Augmented Virtuality Tele-Conferencing. In: I Workshop sobre Realidade Aumentada, Piracicaba, Brasil, Maio, 2004.

[9] PROVIDELO, C.; DEBONZI, D.H.; GAZZIRO, M.A.; QUEIROZ, I.C.A.S.; KIRNER C.; SAITO, J.H. Ambiente Dedicado para Aplicações Educacionais Interativas com Realidade Misturada. In: I Workshop sobre Realidade Aumentada, Piracicaba, Brasil, Maio, 2004.

[10] ARTOOLKIT: http://www.hitl.washington.edu/rese /shared_space. Visitado pela ultima vez em 18/10/2005.

[11] FIALA, M. "ARTag, a Fiducial Marker System Using Digital Techniques," cvpr, pp. 590 - 596, 2005 1EEE Computer Society Conference on Computer Vision and Pattern Recognition (CVPR905) - Volume 2, 2005.

[12] ARTAG: http://www.artag.net/. Visitado pela ultima vez em 27/08/2010.

[13] http://vrmlworks.crispen.org/history.html/. Visitado pela ultima vez em 18/08/2010.

[14] KIRNER, Cláudio; SANTIN, Rafael. Funcionamento e Utilização do Sistema de Autoria Colaborativa com Realidade Aumentada - SACRA. 2010.

[15] KIRNER, C. Mãos Colaborativas em Ambientes de Realidade Misturada. Anais do WRA2004. Universidade Metodista de Piracicaba, 2005.

[16] BERNARDINO A. JUNIOR, Afonso; CARDOSO, Alexandre; LAMOUNIER JR., Edgard. Estudo de Máquinas Elétricas Auxiliado por Técnicas de Realidade Virtual. Universidade Federal de Uberlândia - Faculdade de Engenharia Elétrica.

[17] CUNHA, Kelma Kerly de Carvalho; GUIMARÃES, Marcelo de Paiva. Desenvolvimento de uma Interface Gráfica para o Artoolkit com Aplicação na Área Educacional. WRVA'07 Workshop de Realidade Virtual e Aumentada, Itumbiara 2007.

[18] TRIGO, C. H.; KIRNER, C. Incorporando Colaboração com Realidade Virtual e Aumentada em Ambientes de Educação à Distância. WRVA'07 Workshop de Realidade Virtual e Aumentada, Itumbiara 2007.

[19] BLUEMEL, E.; HINTZE, A.; SCHULZ, T.; SCHUMANN, M.; STUERING, S.. Virtual Environments for the Training of Maintenance and Service Tasks. Proceedings of the 2003 Winter Simulation Conference, vol.2.. The Fairmont New V. $9 \mathrm{~N}^{\circ} 1$, julho, 2011 
Orleans, New Orleans, LA. 7-10 Dec. 2003. p.p. 2001-2007. Digital Object Identifier 10.1109/WSC.2003.1261664, 2003.

[20] BOUD, A. C.; HANIFF, D. J.; BABER, C.; STEINER, S. J.. Virtual Reality and Augmented Reality as a Training Tool for Assembly Tasks. International Conference on Information and Visualization, vol. 4, pp.32-36, 1999.

[21] LI, J.R.; KHOO, L. P.; TOR, S. B. Desktop Virtual Reality for Maintenance Training: an Object Oriented Prototype System (V-REALISM). Computers in Industry vol. 52, p.p. 109-125, 2003.

[22] FEINER, S.; MACINIYRE, B.; SELIGMANN, D. Knowledge based Augmented Reality. Communications of the ACM, Julho, 1993.

[23] COMPORT, AI; MARCHAND, F Chaumette. A real-time tracker for markerless augmented reality. In: Proceedings of the Second IEEE and ACM International Symposium on Mixed and Augmented Reality. ISMAR, pp: 36- 45, 2003. [24] ESPÍNDOLA, D. B; PEREIRA, C.E; HENRIQUES, R.V.B; BOTELHO, S.S. Using Mixed Reality in the Visualization of Maintenance Processes. IFAC Workshop on Advanced Maintenance Engineering, Services and Technology, Lisboa, 2010 .

[25] TIMM, M. I.; ROLIM, Odilon; LONGHI, Magali; FERNANDES, Luis Fernando; EWALD, Denise Grüne; CABRAL JR., Paulo Augusto; FERREIRA FILHO, Raymundo C.M. Gestão de EAD: projeto de infra-estrutura e atividades de planejamento. In: V ESUD Congresso Brasileiro de Ensino Superior à Distância, 2008. [26] FORTE, C; OLIVEIRA, F.; SANTIN, R.; KIRNER, C. Implementação de Laboratórios Virtuais em Realidade Aumentada para Educação à Distância. 5 Workshop de Realidade Virtual e Aumentada. Bauru, 2008.

[27] http://www.youtube.com/user/cesupUFRGS Visitado pela ultima vez em 27/08/2010.

[28] http://www.ckirner.com/sacra/SACRA-2010.zip Visitado pela ultima vez em 07/08/2010. 